\title{
Resuscitative Endovascular Balloon Occlusion of the Aorta is a Risk Factor for Intestinal Necrosis: A Case Report
}

\section{Keitaro Yajima}

Department of Trauma and Emergency Surgery, Saiseikai Yokohama-shi Tobu Hospital https://orcid.org/0000-0002-7938-7097

Shokei Matsumoto ( $\square$ m-shokei@feel.ocn.ne.jp )

Saiseikai Yokohama-shi Tobu Hospital

\section{Motoyasu Yamazaki}

Department of Trauma and Emergency Surgery Saiseikai Yokohama-shi Tobu Hospital

\section{Case report}

Keywords: resuscitative endovascular balloon occlusion of the aorta, complication, intestinal necrosis

Posted Date: November 9th, 2020

DOl: https://doi.org/10.21203/rs.3.rs-102623/v1

License: (a) (1) This work is licensed under a Creative Commons Attribution 4.0 International License. Read Full License 


\section{Abstract}

\section{Background}

Resuscitative endovascular balloon occlusion of the aorta (REBOA) is effective for temporary hemorrhage control and resuscitative effort, as it enhances cerebral and coronary circulation in trauma patients. However, an inappropriate utilization of REBOA leads to critical complications. Placement of the balloon in Zone 2 of the aorta should be avoided as the occlusion restricts the intestinal blood supply leading to fatal complications. There is a scarcity of case reports on complications associated with endovascular balloon occlusion in the literature. Here, we have presented a rare case in which Zone 2 REBOA contributed to an ischemic complication in a trauma patient.

Case presentation

A 50-year-old man with severe trauma, who accidentally got buried under a fallen cement wall, was carried to the nearest hospital. Contrast-enhanced computed tomography showed an unstable pelvic fracture that required hemostatic intervention. Prior to being transferred to another hospital, the patient was treated with endovascular balloon placement. A Zone 2 endovascular balloon placement with resuscitative effort accidentally led to insufficient abdominal blood flow and he developed extensive intestinal necrosis. Following surgical intervention, the patient was resuscitated; however, he developed partial intestinal necrosis and was subsequently managed with surgical intestinal resection.

Conclusions

Blood supply to the abdominal organs should be considered when deploying the balloon. Further, balloon positioning, aortic occlusion time, and inflation volume should be carefully considered to avoid ischemic complications.

\section{Background}

Resuscitative endovascular balloon occlusion of the aorta (REBOA) is effective for temporary hemorrhage control and resuscitative effort, as it enhances cerebral and coronary circulation in trauma patients; however, it can cause critical complications [1]. In particular, it is necessary to pay attention to ischemic complications. Therefore, placement of the balloon at aortic Zone 2 should always be avoided to preserve blood supply to the abdominal organs. However, limited case reports have described complications related to gastrointestinal hypoperfusion. We have presented a rare case in which Zone 2 REBOA contributed to an ischemic complication in a trauma patient.

\section{Case Presentation}

A 50-year-old man, in a state of severe shock and unconsciousness, presented to the first attending hospital after accidentally getting buried under a cement wall. At the hospital, he was immediately 
intubated and initial resuscitation was started. After his vital signs stabilized, contrast-enhanced computed tomography (CECT) was performed and showed left hemopneumothorax, left lung contusion, and an unstable pelvic fracture. His condition became unstable again immediately after the CECT.

The attending physician decided to transfer the patient to our hospital for surgical management. A REBOA catheter was inserted via the right femoral artery and positioned using the nipples as the external landmark for Zone 1 placement; the balloon was inflated to a diameter of $30 \mathrm{~mm}$ (Table 1). Prior to the patient's transfer, no image was taken to confirm the balloon position at Zone 1. The patient's blood pressure immediately stabilized, and he was transported by ground ambulance for surgical management. He was administered 10 units of red blood cells during transportation. Upon arrival at the hospital, we observed that the balloon was not secured at its position.

As our hybrid trauma bay is equipped for fluoroscopy, computed tomography, and surgery, we initially checked the location of the balloon using fluoroscopy. We confirmed that the center of the inflated balloon was located in Zone 2 (the L1 vertebral level) and subsequently relocated it to Zone 1 . The positional relationship between the REBOA balloon and splanchnic artery was confirmed via radiographics (Figure 1). A massive transfusion protocol was also initiated. The patient's blood pressure increased as we performed peritoneal pelvic packing, and we fully deflated the balloon. The total occlusion time was $82 \mathrm{~min}$. Subsequently, transcatheter arterial embolization was performed for the severe pelvic fracture because the patient's condition was unstable. The patient's vital signs improved markedly after successful embolization. However, his abdomen became tense and tender, and the tidal volume decreased. These symptoms suggested abdominal compartment syndrome.

The patient underwent urgent decompressive laparotomy for abdominal compartment syndrome. Ischemic necrosis was observed in the ascending colon, half of the transverse colon, and $110 \mathrm{~cm}$ of the terminal ileum (Figure 2). The ischemic lesion was resected without primary anastomosis. Given that there are many risk factors for abdominal compartment syndrome such as aggressive resuscitation, ischemia-reperfusion injury, visceral swelling, intra-peritoneal packing, and coagulopathy, an open abdominal management was performed with a vacuum-assisted closure. Moreover, this procedure was necessary to recheck the bowel perfusion. The pathological findings of the resected intestine confirmed the absence of embolization in the vessels.

We performed ileocolonic anastomosis on Day 1 after the first operation, followed by definitive closure on Day 6. The patient's postoperative course was complicated by acute kidney injury (AKI). He required temporary renal replacement therapy that ended on Day 11, as his AKI condition improved. After he gained consciousness, bilateral paraplegia was observed on Day 20. Magnetic resonance imaging revealed a spinal cord injury (SCl) at the thoracic vertebral level (T11) and a vertebral fracture. The bilateral paraplegia did not recover. He was discharged from the hospital to a neurologic rehabilitation facility on post-operative day 34 .

\section{Discussion And Conclusions}


Recently, REBOA has emerged as an effective method for temporary control of hemorrhage and maintaining perfusion of the heart and brain in patients with severe torso trauma. However, the practical instructions for REBOA are unclear and the complications arising from inappropriate balloon use could be lethal. Visceral ischemia is a potential critical complication of REBOA [1]. The anatomical zone concept was created for REBOA placement considering visceral ischemia and the bleeding area [2]. REBOA should be safely and accurately positioned in the intended anatomical zone. Zone 1 placement (distal to the left subclavian origin; proximal to the celiac axis) is for solid organ and/or abdominal vascular injuries, and Zone 3 placement (distal to the renal arteries; proximal to the aortic bifurcation) is for isolated pelvic injuries, whereas, Zone 2 placement (visceral segment of the aorta) should be avoided due to the possibility of mesenteric ischemia. However, limited cases of ischemic complications caused by Zone 2 occlusion have been reported.

Although there are several types of acute gastrointestinal ischemia, the anatomical blood flow suggests that ischemia caused by REBOA Zone 2 placement is like ischemia associated with superior mesenteric artery (SMA) occlusion. The SMA originates from the anterior surface of the aorta around the L1 vertebral level [3]. In this case, the center of the REBOA balloon was identified by fluoroscopy at the L1 vertebral level, and the ischemic bowel area corresponded with the SMA perfusion area. These facts support the simple occlusion of the SMA as the potential etiology of the ischemic change in the bowel.

In this case, the most likely cause of the observed ischemia was the incorrect deployment of the REBOA balloon at Zone 2 by the physician at the previous hospital. This may have occurred due to technical misplacement of the balloon. In particular, the REBOA balloon was placed using the nipples as the external landmarks. However, the sternal notch and umbilicus are the most common REBOA placement landmarks for Zone 1 and Zone 3, respectively [4-6]. Although REBOA using a landmark is a simple and easy method, it is inaccurate because of non-image-guided placement. Several methods for balloon positioning have been proposed; yet, appropriate REBOA placement is a challenging procedure [6]. A novel procedure is needed for REBOA placement. Another possible cause of the incorrect balloon deployment could involve catheter migration and movement of the balloon from Zone 1 to Zone 2, owing to an incompletely secured balloon sheath. The balloon can easily shift downward because of the aortic pressure. Therefore, to avoid inadvertent Zone 2 placement, it is important to check the balloon position under fluoroscopy; the balloon sheath must be completely secured.

Despite the correct deployment of the balloon at Zone 1, the visceral blood flow below the balloon may not supply sufficient oxygen to meet the gastrointestinal demand. Whereas Zone 1 aortic occlusion does not completely impede the visceral blood flow owing to multiple pathways of collateralization [7], Zone 1 REBOA inflation decreased the SMA blood flow by more than $80 \%$ in an animal study [8]. Thus, REBOA Zone 1 placement may cause "non-occlusive" mesenteric ischemia, a type of acute gastrointestinal ischemia.

To avoid visceral ischemic complications, it is important to consider not only balloon positioning but also the duration of balloon inflation. In an animal study, a 60-min occlusion resulted in significant organ 
damage [9]. Other animal studies showed that ischemic visceral damage owing to REBOA occlusion was time-dependent $[8,10]$. In this case, an 82-min occlusion period could have certainly led to visceral ischemia. Furthermore, the balloon inflation volume should also be considered. The blood flow below the balloon is negatively associated with the degree of aortic occlusion [11]. Therefore, the inflation time should be minimized and an appropriate partial-REBOA may be useful.

In addition to gastrointestinal ischemia, REBOA may cause ischemia-reperfusion injuries in other organs. Animal studies have reported serum marker and histopathological evidence of renal injury and SCI [12]. In our case, the patient developed AKI, which warranted temporary renal replacement therapy, and SCI resulting in bilateral paraplegia. These complications may be related to an ischemia-reperfusion injury caused by REBOA. However, the contrast material or a vertebral fracture may also have been the cause.

In conclusion, REBOA may be useful for non-compressible torso hemorrhage, although it also carries a potential risk of life-threating complications. We should, therefore, remain vigilant in detecting ischemic organ damage. To avoid serious complications, the priority must be to ensure the appropriate placement zone, achieve the desired inflation volume/duration, properly secure the catheter for transport, and optimize the use of partial or intermittent REBOA, when possible.

\section{Abbreviations}

REBOA=Resuscitative endovascular balloon occlusion of the aorta

CECT $=$ contrast-enhanced computed tomography

AKI=acute kidney injury

$\mathrm{SCl}=$ spinal cord injury

$\mathrm{SMA}=$ superior mesenteric artery

\section{Declarations}

\section{Ethics approval and consent to participate}

Informed consent was obtained for the case report, images, and publication. The study protocol was approved by the Ethics Committee of Saiseikai Yokohama-shi Tobu Hospital (No. 20190208).

\section{Consent for publication}

See consent form.

\section{Availability of data materials}


Data sharing is not applicable to this article as no datasets were generated or analyzed during the current study.

\section{Competing interests}

The authors declare that they have no competing interests.

\section{Funding}

Not applicable.

\section{Authors' contributions}

K.Y., and S.M. participated in the study design. K.Y., S.M. and M.Y. participated in drafting the article.

\section{Acknowledgments}

Not applicable.

\section{References}

[1] Davidson AJ, Russo RM, Reva VA, Brenner ML, Moore LJ, Ball C, et al. The pitfalls of resuscitative endovascular balloon occlusion of the aorta: risk factors and mitigation strategies. J Trauma Acute Care Surg. 2018;84(1):192-202. doi:10.1097/TA.0000000000001711

[2] Stannard A, Eliason JL, Rasmussen TE. Resuscitative endovascular balloon occlusion of the aorta (REBOA) as an adjunct for hemorrhagic shock. J Trauma Acute Care Surg. 2011;71(6):1869-72. doi:10.1097/TA.0b013e31823fe90c

[3] Moore KL, Dalley AF. Clinically Oriented ANATOMY 5th edition. StatPearls Pub/ Treasure Is/. 206AD:272-276. doi: 10.1097/01.shk.0000222896.39363.fa

[4] Linnebur M, Inaba K, Haltmeier T, Rasmussen TE, Smith J, Mendelsberg R, et al. Emergent non-imageguided resuscitative endovascular balloon occlusion of the aorta (REBOA) catheter placement: a cadaverbased study. J Trauma Acute Care Surg. 2016;81(3):453-7. doi:10.1097/TA.0000000000001106

[5] Okada Y, Narumiya H, Ishi W, liduka R. Anatomical landmarks for safely implementing resuscitative balloon occlusion of the aorta (REBOA) in zone 1 without fluoroscopy. Scand J Trauma Resusc Emerg Med. 2017;25(1):63. doi:10.1186/s13049-017-0411-z

[6] Matsumoto S, Funabiki T, Kazamaki T, Orita T, Sekine K, Yamazaki M, et al. Placement accuracy of resuscitative endovascular occlusion balloon into the target zone with external measurement. Trauma Surg Acute Care Open. 2020;5(1):1-6. doi:10.1136/tsaco-2020-000443 
[7] Wasicek PJ, Shanmuganathan K, Teeter WA, Gamble WB, Hu P, Stein DM, et al. Assessment of blood flow patterns distal to aortic occlusion using CT in patients with resuscitative endovascular balloon occlusion of the aorta. J Am Coll Surg. 2018;226(3):294-308. doi:10.1016/j.jamcollsurg.2017.12.005

[8] Hoehn MR, Teeter WA, Morrison JJ, Gamble WB, Hu P, Stein DM, et al. Aortic branch vessel flow during resuscitative endovascular balloon occlusion of the aorta. J Trauma Acute Care Surg. 2019;86(1):79-85. doi:10.1097/TA.0000000000002075

[9] Reva VA, Matsumura Y, Hörer T, Sveklov DA, Denisov AV, Telickiy SY, et al. Resuscitative endovascular balloon occlusion of the aorta: what is the optimum occlusion time in an ovine model of hemorrhagic shock? Eur J Trauma Emerg Surg. 2018;44(4):511-8. doi:10.1007/s00068-016-0732-z

[10] Markov NP, Percival TJ, Morrison JJ, Ross JD, Scott DJ, Spencer JR, et al. Physiologic tolerance of descending thoracic aortic balloon occlusion in a swine model of hemorrhagic shock. Surgery. 2013;153(6):848-56. doi:10.1016/j.surg.2012.12.001

[11] Reva VA, Matsumura Y, Samokhvalov IM, Pochtarnik AA, Zheleznyak IS, Mikhailovskaya E, et al. Defining degree of aortic occlusion for partial-REBOA: a computed tomography study on large animals. Injury. 2018;49(6):1058-63. doi:10.1016/j.injury.2018.04.021

[12] Eliason JL, Myers DD, Ghosh A, Morrison JJ, Mathues AR, Durham L, et al. Resuscitative endovascular balloon occlusion of the aorta (REBOA): zone I balloon occlusion time affects spinal cord injury in the nonhuman primate model. Ann Surg. 2019; [Epub ahead of print]. doi:10.1097/SLA.0000000000003408. [Epub ahead of print]

\section{Tables}




\begin{tabular}{|c|c|c|c|c|c|c|c|}
\hline \multirow[t]{2}{*}{ Time } & \multirow[t]{2}{*}{ Events } & \multicolumn{2}{|c|}{$\begin{array}{l}\text { REBOA } \\
\text { deployment }\end{array}$} & \multirow{2}{*}{$\begin{array}{l}\text { SBP } \\
(\mathrm{mmHg})\end{array}$} & \multirow{2}{*}{$\begin{array}{l}\mathrm{HR} \\
\text { (bpm) }\end{array}$} & \multirow{2}{*}{$\begin{array}{l}\text { Lac } \\
(\mathrm{mg} / \mathrm{dL})\end{array}$} & \multirow[t]{2}{*}{$\begin{array}{l}\text { Additional } \\
\text { notes }\end{array}$} \\
\hline & & Zone & $\begin{array}{l}\text { Diameter } \\
(\mathrm{mm})\end{array}$ & & & & \\
\hline $16: 20$ & $\begin{array}{l}\text { Presented to } \\
\text { the first } \\
\text { hospital }\end{array}$ & - & - & Unmeasurable & 146 & & $\begin{array}{l}\text { Initial } \\
\text { resuscitation } \\
\text { Intubated. }\end{array}$ \\
\hline $16: 26$ & CECT & - & - & 60 & 130 & & $\begin{array}{l}\text { Right chest } \\
\text { tube }\end{array}$ \\
\hline $19: 20$ & $\begin{array}{l}\text { REBOA was } \\
\text { deployed } \\
\text { (30-mm balloon } \\
\text { diameter) }\end{array}$ & 1 & 30 & 88 & 121 & & $\begin{array}{l}\text { Blood } \\
\text { transfusion } \\
\text { started. }\end{array}$ \\
\hline $20: 10$ & $\begin{array}{l}\text { Transferred to } \\
\text { our hospital }\end{array}$ & 2 & 30 & 89 & 111 & 225 & $\begin{array}{l}\text { RBC } 10 \mathrm{U}, \\
\text { ECF } 2000 \mathrm{ml} \\
\text { infused }\end{array}$ \\
\hline $20: 28$ & $\begin{array}{l}\text { REBOA } \\
\text { repositioning }\end{array}$ & $2 \otimes 1$ & 30 & 107 & 99 & & $\begin{array}{l}\text { REBOA was } \\
\text { located in } \\
\text { Zone } 2 \\
\text { MTP } \\
\text { initiated } \\
\text { PPP started }\end{array}$ \\
\hline $20: 35$ & $\begin{array}{l}\text { REBOA balloon } \\
\text { deflate } \\
\text { (15-mm balloon } \\
\text { diameter) }\end{array}$ & 1 & 15 & 107 & 111 & & PPP finished \\
\hline $20: 50$ & $\begin{array}{l}\text { REBOA } \\
\text { repositioning } \\
\text { (full deflation) }\end{array}$ & $1 \otimes 3$ & 0 & 103 & 113 & & CECT \\
\hline $21: 00$ & IR started & 3 & 0 & 109 & 109 & 179 & $\begin{array}{l}\text { TAE for } \\
\text { pelvic } \\
\text { fracture }\end{array}$ \\
\hline $22: 20$ & $\begin{array}{l}\text { Decompressive } \\
\text { laparotomy }\end{array}$ & 3 & 0 & 119 & 103 & 174 & $\begin{array}{l}\text { RBC } 28 \mathrm{U}, \\
\text { FFP } 34 \text { U, PC } \\
20 \text { U, ECF } \\
4320 \mathrm{ml} \\
\text { ACS }\end{array}$ \\
\hline
\end{tabular}


$\mathrm{SBP}=$ systolic blood pressure, $\mathrm{HR}=$ Heart rate, $\mathrm{RR}=$ Respiratory rate, $\mathrm{ROSC}=$ Return of spontaneous circulation, CECT=contrast-enhanced computed tomography, MTP=Massive transfusion protocol, PPP=Preperitoneal pelvic packing, REBOA=Resuscitative endovascular balloon occlusion of the aorta, $\mathrm{IR}=$ Interventional radiology, $\mathrm{TAE}=$ Transcatheter arterial embolization, $\mathrm{ECF}=$ Extracellular fluid, ACS=abdominal compartment syndrome

Vital signs are unmeasurable due to severe shock when the patient arrived at the first hospital (line 1). REBOA was deployed (30-mm balloon diameter by inflation) for the severe trauma, and transfusion had started during transfer. REBOA was repositioned in Zone 1, PPP and IR was performed after arrival at our hospital. Owing to these procedures, the patient was becoming stable and the balloon could be deflated. However, the patient suffered from abdominal compartment syndrome and therefore, finally, decompressive laparotomy was performed.

\section{Figures}

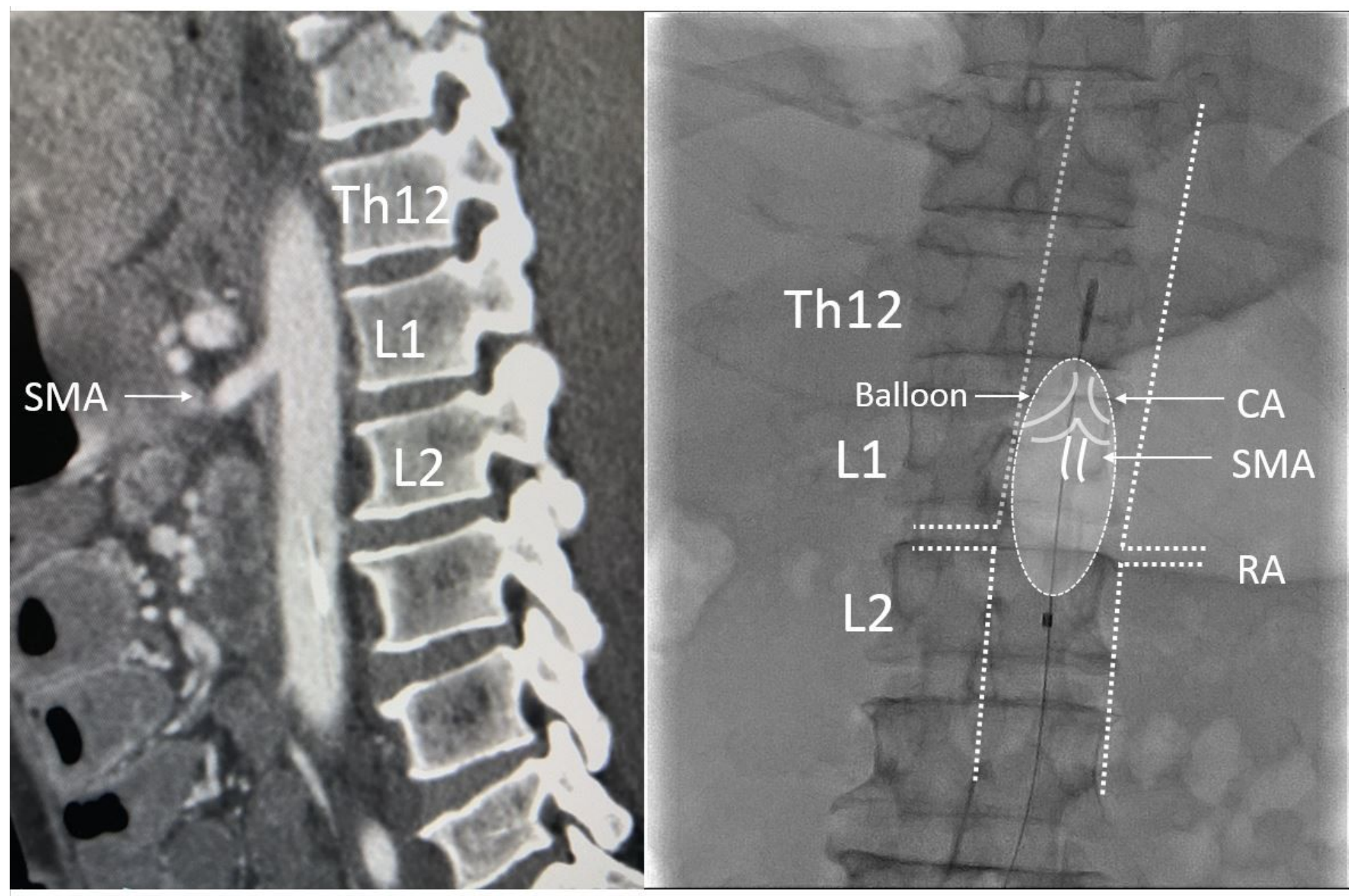

\section{Figure 1}

Positional relationship between the REBOA balloon (RESCUE BALLOON-ER; Tokai Medical Products, Aichi, Japan) and the splanchnic artery Computed tomography (CT) showing the positional relationship 
between the REBOA balloon and the splanchnic artery. The aorta and its branches on fluoroscopy were constructed from the CT scan. Fluoroscopy revealing that the center of the balloon ,between the radiopaque markers, was located in Zone 2 (L1 vertebral level). ${ }^{*} \mathrm{CA}=$ Celiac artery, ${ }^{*} \mathrm{SMA}=$ Superior mesenteric artery, ${ }^{*} \mathrm{RA}=$ Renal artery

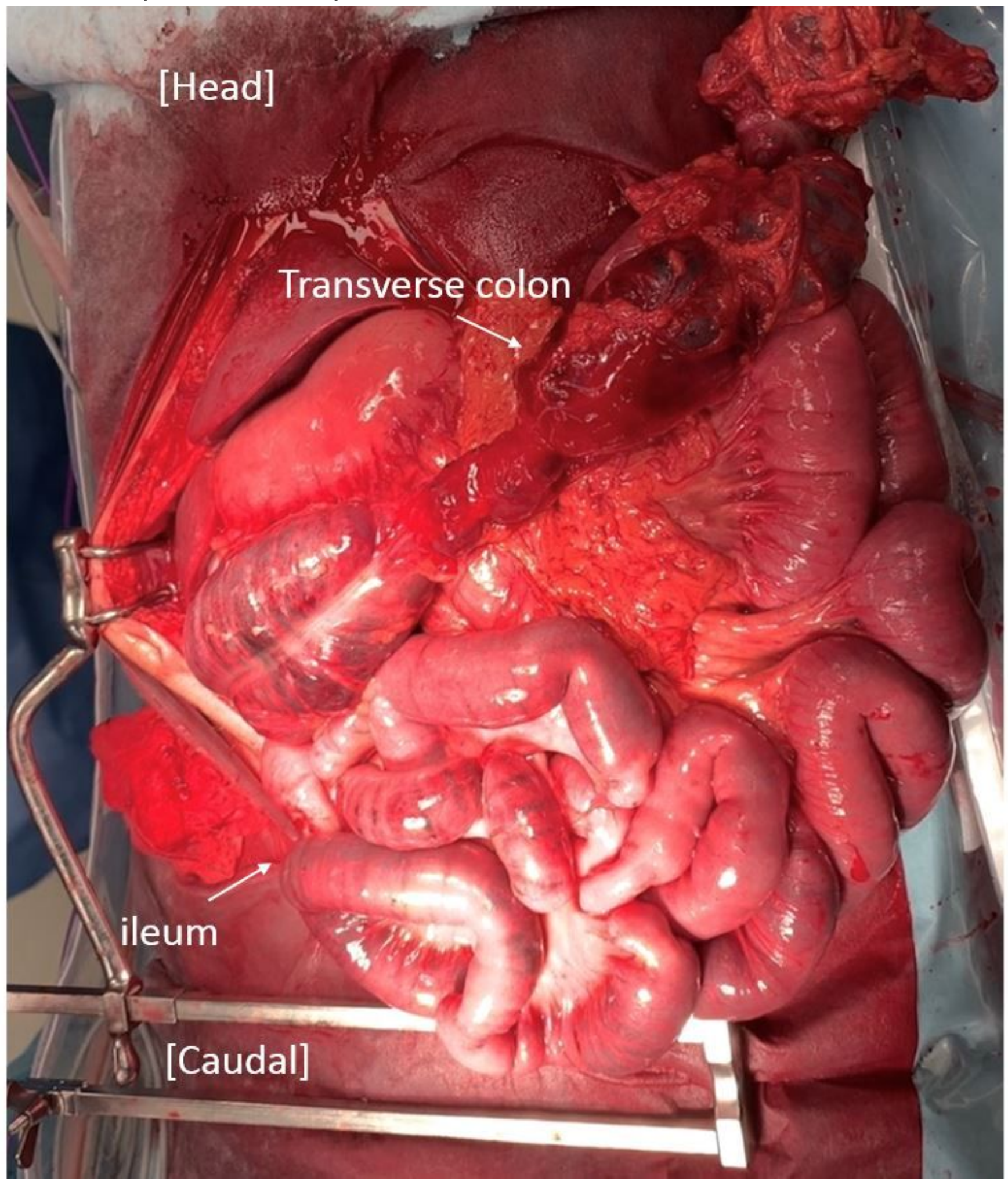

Figure 2 
Ischemic necrosis after resuscitative effort Ischemic necrosis was observed in the ascending colon, half of the transverse colon, and $110 \mathrm{~cm}$ of the terminal ileum 